\title{
USING MIND MAP IN TEACHING THE SUBJECT OF AGGREGATE STATES OF MATTER
}

\section{Ganiev A.G.}

Candidate of Physical and Mathematical Sciences, Associate Professor, Director of Shakhrisabz branch of Tashkent State Pedagogical University

\section{ABSTRACT}

The article discusses the factors that improve people's intellectual abilities. To do this, it provides information about the physiology of the human brain and the mechanism by which the information it perceives is stored in memory. To develop students' 'creative thinking' skills, it is necessary to develop their 'imagination' skills. The imagination perceives information from the mind. "Indirect perception" is important for imagining physical processes. Data visualization is important for understanding processes. To do this, use the "Mind Map", which is an effective way to perform such a task. The article describes the opportunities for students to develop "creative thinking" skills in the teaching of "Aggregate states of matter." For the first time, "Aggregate Cases" are being published.

Not only it stores a lot of information about physical processes, but it also helps students develop "full thinking" skills by activating the cerebral hemispheres.

KEYWORDS:- Technology, Thinking, Genetics, Education, Environment, Brain,

\section{INTRODUCTION}

People have different thinking abilities. What is the reason for this? Genetics (human biological nature) or environment and upbringing?

In general, the genetic code passed down from generation to generation plays an important role in the formation of the human intellect [1]. The DNA is irreversible and unique, and only the first steps are being taken to change it. In 2020 Nobel Prize was given to E. Sherpante and Dj. Dudna for their discoveries on change in Chemistry for DNA
Testing of Genome and Plant DNA [2]. At the same time, scholars acknowledge the unique role of education in the formation of the individual. According to them, the use of innovative teaching methods can improve students' thinking skills and improve their memory dozens of times. Thus, although genetics plays an important role in a person's intellectual potential, the influence of the environment in which he or she grows up is also significant [3].

Are people's brains the same or different? Everyone's brain consists of

The cerebral cortex, the right and left 
hemispheres of the brain. American neurophysiologist Sperry Roger was awarded the Semi-Nobel Prize in 1981 for his "discovery of a connection to the functional specialties of the cerebral hemispheres."

The left hemisphere of the brain controls the right side of the body. Mechanisms of logical thinking have well-developed in them.

The right hemisphere of the brain controls the left side of the body. Mechanism of figurative thinking have developed well in them, which create non-standard ideas.

What changes take place in the human brain during education? Neurobiologist and biochemist Erek Kandel (winner of the 2006 Nobel Prize) has shown that the "transmitter" (neuron) of the human brain changes during education. Even the smallest detail can lead to changes in the structure of the neuron [4].

Neuron fibers open specific channels of action. These canals are like "trails". There are no two people in the world who have the same "paths". Different parts of the human brain develop differently. No matter what we do or learn throughout our lives, it affects the shape and appearance of the neurons in our brain. In other words, it changes its "trails" [3].

Subsequent data moves from the previously opened channel, and over time, this channel becomes dominant and becomes the main path. This means that all similar information is stored in this "main path" (the trace in the brain), that is, in memory. It's about understanding information. This is how comparing new information with what is in the brain ("comparative learning"). If there were no such main path, and if there was a separate path for each piece of information, the brain would be full of paths.

Comparative learning is the most effective method in the education system. This feature is unique to man and is one of the main factors that make him superior to the computer. Various "models" have been devised to use this method. For example, in physics, such concepts as "material point", "absolutely solid", "ideal gas", "point charge" can be cited as such a model.

In his works, al-Beruni expressed his views on the universality of the laws of nature. G. Galileo, on the other hand, saw the shadow of the mountains on the Moon and found experimentally that the laws of shadow formation are relevant to the heliocentric system.

E. Rutherford, who knew the motion of planets in a heliocentric system, analyzed the experimental results and proposed a "planetary model" of the atom.

Based on Feynman's diagram (quantum electrodynamics), which shows that the interaction of charged particles in electrodynamics is carried out by means of pharaohs, it is said that strong interactions between quarks also occur by means of similar gluons (G) (quantum chromodynamics). concluded.

There are many examples of such unification in physics.

Although "comparative learning" is a good way to activate the brain, it only helps to identify differences in comparisons and keep them in memory. That is, it does not develop "imagination" and "creative thinking" skills. In order to "imagine", the brain gets information from the mind.

Intelligence - the process by which information is received and analyzed in the brain. It allows us to evaluate things and events in the external world and act accordingly.

Intelligence, which is based on information received through sensory channels about things or real events happening around them, is called 


\section{direct intelligence.}

Perception of information transmitted in written, oral and other (visual) forms is called indirect perception. [5]

At the same time, the question of how intuition affects perception and existing experiences has been in the focus of scholars since ancient times. More precisely, does all information about the external world come through our senses, or is a certain part of it transmitted to perception through the "mind independent of the external world"?

This issue is especially important for the "natural sciences" - the laws of nature, the laws of which are not formed in human activity. This is because most of the knowledge acquired in the study of physics, chemistry and biology, which falls into this category, is obtained "indirectly". In other words, they are obtained through various technical means, computers, information prepared by others, and improved by analyzing their knowledge and experience.

Experience has shown that some of the information we receive for perception has nothing to do with existence. According to English philosopher and psychologist Dj. Locke's concept of "nativis" vertical (nature), perception is based on the "mind" given to man by nature, more precisely, on the information passed through our DNA. Only this information is activated by experience, knowledge, thinking. Our brain stores information from the outside world in the form of "memory traces". They, in turn, have a direct impact on perception, the nature and the meaning of the "imagined" images.

Imagination is the remembrance of things and events, situations, images of reality.

1) Passive or forced imagining - unforeseen events, travel, landscapes, landscapes, communication, imaginary images can occur independently of human will.

2) Active or voluntary imagination - an image based on information that a person perceives and already exists in the brain. Creative imagination is also active imagination. At the same time, "passive imagination" plays an important role in the "creative process".

\section{Results. Intelligence map "Aggregate states of matter".}

Below, we explore opportunities for students to develop "creative thinking" skills as they move on to the topic of aggregate states of matter.

\section{The energy of the interaction of molecules depends on the distance between them.}

Molecules interact, which means that the potential energy of the interaction is Ep. The figure shows a potential line where the potential energy of an intermolecular interaction depends on the distance between them [6-8].

The repulsive potential energies of molecules are positive, the potential energies of gravity are negative. The potential energies of the molecules at infinite distances from each other are zero, that is, they do not interact. As they get closer to each other, the potential energies of the interaction decrease, that is, they accept negative values smaller than zero, and at $r=r 0$ da $(F=0)$ to their smallest Epmin value, the stability of the molecules reaches equilibrium. The subsequent convergence of the molecules is due to the work done by external forces against the repulsive forces, leading to a sharp increase in the potential energy of the molecules. The ABC part of the potential line is called the potential medium; Epmin is the depth of the potential gap. However, because molecules are in constant motion, they also have a certain kinetic energy. The minimum value of the potential energy of intermolecular interactions Epmin and the average value of the kinetic energy of chaotic motion of molecules $\mathrm{E}_{-} \mathrm{k}$ determine the 
aggregate states of substances:

1. $\mathrm{E}_{-} \mathrm{k}>>\mathrm{Ep}$ min - the substance is in the gaseous state;

2. $\mathrm{E}_{-} \mathrm{k}<<\mathrm{Ep} \min$ - the substance is in a solid state;

3. $\mathrm{E}_{-} \mathrm{k} \approx \mathrm{Ep} \min$ - the substance is in a liquid state;

Aggregate states of a substance (Latin aggrego-combine) are states of the same substance in different intervals (intervals) of temperature and pressure.

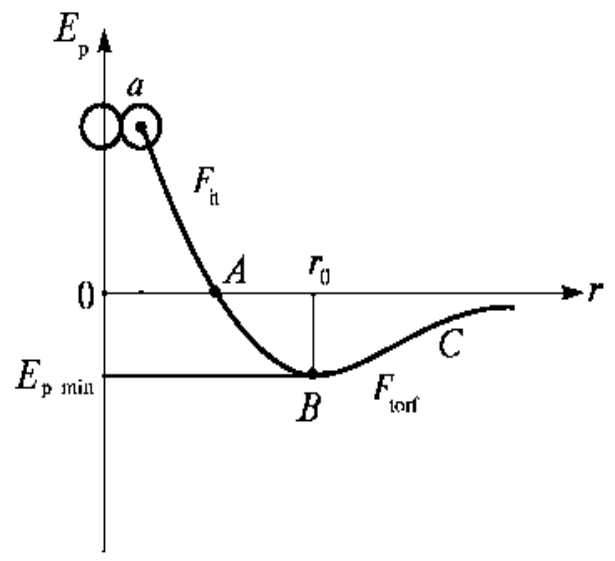

\section{1-figure}

Aggregate states are gaseous (Greek, chaos, disorder), liquid and solid states of matter.

Ice, water, and water vapor are the most common examples of a substance in three different aggregate states in our daily lives. Invisible water vapor is always

around us. Water in the range from $00 \mathrm{C}$ to 1000C, and ice at temperatures below 00C. At normal atmospheric pressure and temperatures above 1000C, water molecules are only gaseous. Water, ice and water vapor are substances with the chemical formula $\mathrm{H} 2 \mathrm{O}$.

Most substances are normally in a single aggregate state. For example, under normal conditions, oxygen is a gas, iron is a solid, and so on.

Each substance can be in different aggregate states. In doing so, they differ from each other in the location and motion of the molecules.

Gaseous state of matter. $\mathrm{E}_{-} \mathrm{k}_{\mathrm{k}}>\mathrm{Ep}$ min, matter is in a gaseous state, the molecules move at very high speeds and are at great distances from each other relative to their size. The force of attraction between the molecules is almost zero. That's why they move freely and take up the space allotted to them; they can be compressed as much as they want. The pressure of the gas is the result of the molecules hitting the vessel wall. At these shocks, impulses are given to the walls and the resulting gas pressure is created.

Liquid state of matter. The molecules of these aggregates are more strongly bound to each other. They oscillate mainly around a certain state of equilibrium. The repulsive force between the molecules is so great that it is almost impossible to squeeze the liquids. However, when a liquid is heated, the kinetic energies of the molecules increase and the gravitational forces can break off (evaporate). The main property of a liquid is its fluidity. The liquid flows well and takes the form of any container in which it is poured.

Solid state of matter. This aggregate state of matter, unlike previous states, has a constant shape and volume. The molecules are located at the ends of the crystal lattice and are tightly bound to each other. The forces of gravity are so great that a molecule cannot move far from its neighbor, but can only oscillate around its equilibrium position. The shape and properties of a solid can also vary depending on the location of the molecules in the crystal lattice. In nature, amorphous solids are found along with crystalline solids. The amorphous state of solids is an unstable state in which the molecules are arranged randomly. 
We use the Mind Map to visualize the processes that take place in the aggregate states of matter. [9-11]

The "mind map" activates the cerebral hemispheres and helps to develop the ability to "think fully."[12] It is particularly effective in the study of physics, where there is a great need for indirect perception of information [13-16].

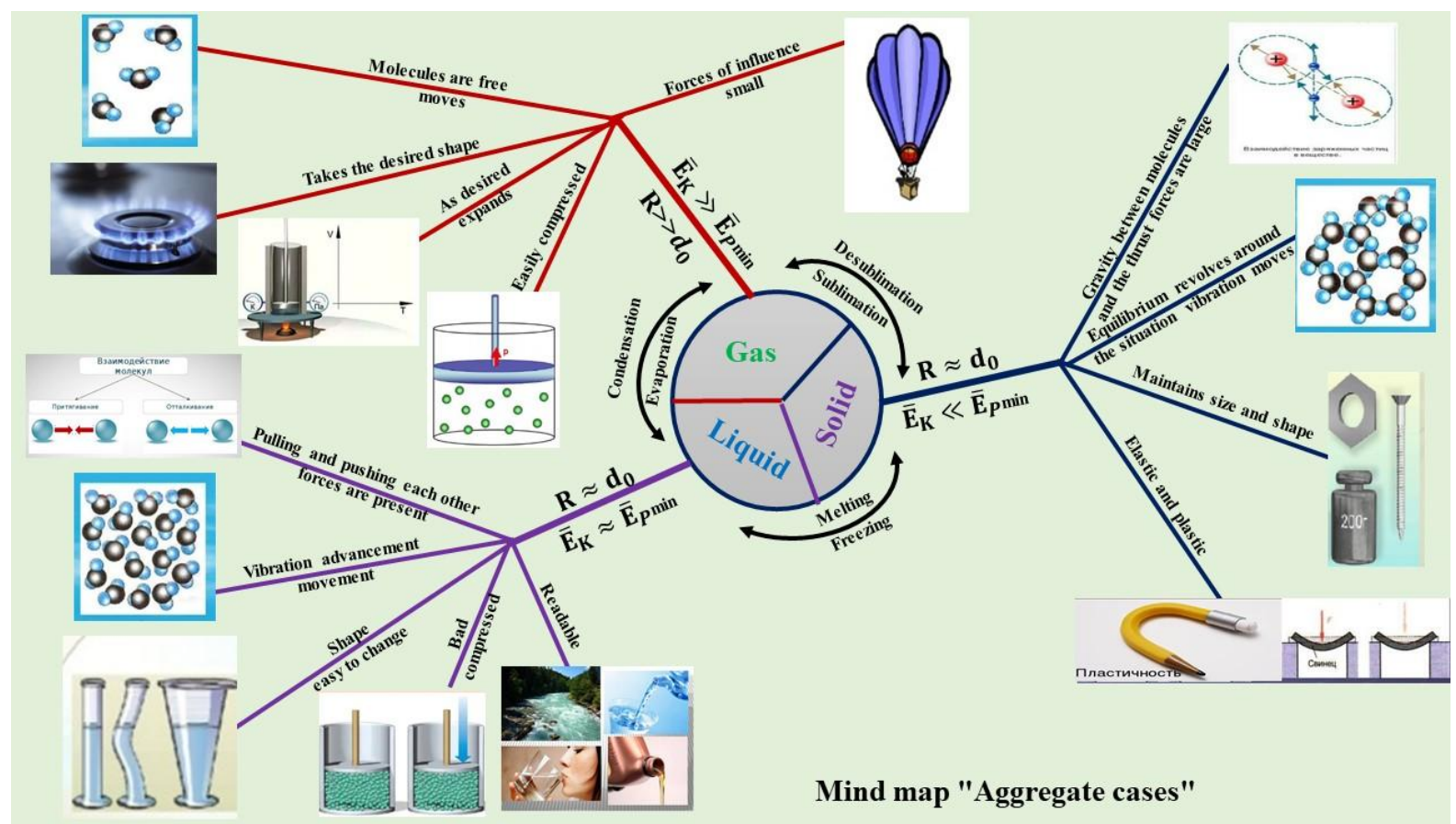

Switch from one aggregate state to another. Under certain conditions, a substance passes from one state to another. These transitions take place through six processes. (see Mind map).

The transition of a substance from a solid (crystalline) state to a liquid state is called melting. The opposite is called crystallization or solidification. For example, melting is the melting of ice, and the reverse is the freezing of water.

The transition of a substance from a liquid to a gaseous state is called evaporation, and vice versa. For example, evaporation is the

\section{2-figure}

evaporation of water, and vice versa, the evaporation of air vapor into dew.

The transition of a substance from a solid state to a gaseous state (without a liquid state) is called sublimation, and vice versa, and the transition of a gas to a solid state is called desublimation. For example, graphite is converted directly to a gaseous state. Dry ice (a solid oxide of $\mathrm{CO} 2$ ) also converts directly to a gaseous state without being liquefied. The same thing happens with naphthalene (we smell naphthalene).

This mind map visualizes all the information about the aggregate states and transitions of 
substances and encourages students to "imagine" these processes. This, in turn, helps them to develop the skills of "imagination", "creative thinking" and, consequently, "full thinking".

\section{Conclusion}

- Although genetics plays an important role in a person's intellectual potential, the impact of the environment in which he grows up is also significant. This means that student intelligence can be improved through the use of productive teaching methods.

- To better understand the processes that take place, it is necessary to imagine them. For physical processes, this is very important, as is the "indirect perception" of information.

- Visualization is important for data perception. The most productive way to accomplish this task is the "Mind Map".

- The article provides a mind map of "Aggregate states". It contains information about the state of matter and its transition processes.

- In addition, the "Aggregate Cases" mind map encourages students to "imagine", "think creatively" and therefore "think fully" and helps them develop skills in this area.

\section{REFERENCES}

1. RS Nemov "Psychology" book 3. Moscow. Vlados. 2005 56-62 p.

2. Laureates of the Nobel Prize in Chemistry in 2020 Nur-Sultan. Kazinform 2020.

3. E. de Bono Serious creative thinking. Mn. Ltd. "Potpourri" 2005 20-80 pages.

4. E.R. Kandel. In Search of Memory / translated by P. Petrov-M. Astrel. 2012.736 p.

5. R.S. Nemov. "Psychology" book1. Moscow. Vlados. 2005 (194-196 pages), (263-265 pages).

6. Ganiev AG and others Physics 10. Tashkent. Niso Polygraph 2017. (pages 173-176).

7. Patrik Fullich Physics., Heinemann Advanced Science 1994 y (p.497-500).

8. R. Feynman et al. Feynnan's lectures on physics M. "MIR" 1977 (38-55 pages).

9. T. Busen "Mind Maps" Moscow. "Mann. Ivanov and Ferver "2019, pp. 25-90.

10. www.imind map.com.

11. www.world mind mapping cannol. com.

12. Zdenek M. Development of the right hemisphere. Potpourri Publishing House, 2004

13. Ganiev A. G. S. N. Tashev THE ROLE OF "IMAGINATION" IN THE PROCESS OF "CREATIVE THINKING". DEVELOPING STUDENTS' "IMAGINATION" AND "CREATIVE THINKING" SKILSS IN TEACHING PHYSICS 3569-3575 202158 (1) PSYCHOLOGY AND EDUCATION.

14. A.G. Ganiev, (2021). Developing Students' 'Creative Thinking' Skills In The Course of 'Law Of Conservation'. The American Journal of Social Science and Education Innovations, 3(03), 564-573.

15. G. Ganiev, Z. Sh. Abdunazarova, Biophysics of Brain Activity. Brain Activity in The Development of "Creative Thinking" "Mind Map", Turkish Journal of Computer and Mathematics Education, PP 1 - 6 e-ISSN 1309-4653; 